\title{
COOPERATIVE EFFECT OF HUMAN PAPILLOMAVIRUS TYPE 18 E5, E6 E7 ONCOPROTEINS IN PROMOTING CELL PROLIFERATION, MIGRATION, INVASION AND IN MODULATING CELLULAR REDOX STATE
}

\section{Jimena Hochmann ${ }^{1}$ : Felipe Parietti ${ }^{1}$, Jennyfer Martinez ${ }^{2}$ : Ana Clara Lopez ${ }^{3}$ : Mara Carreño ${ }^{3}$ Celia Quijano ${ }^{2}$, Laura}

Sichero ${ }^{4}$, Matías Möller ${ }^{3}$, Santiago Mirazo ${ }^{1}$, Juan Arbiza ${ }^{1}$

(1) Sección Virología, Facultad de Ciencias, Universidad de la República (UDELAR). Montevideo-Uruguay.(2) Centro de Investigaciones Biomédicas (CEINBIO and Departamento de Bioquímica, Facultad de Medicina, Universidad de la República (UDELAR). Montevideo-Uruguay. (3) Laboratorio de Fisicoquímica Biológica, Facultad de Ciencias,

Universidad de la República (UDELAR). Montevideo-Uruguay, (4) Center for Translational Research in Oncology, Instituto do Cancer do Estado de São Paulo. Hospital das Clinicas da Faculdade de Medicina da Universidade de São Paulo, São Paulo, Brazil

\section{BACKGROUND}

High-risk human papillomaviruses (HR-HPVs) are the etiological agents of cervical cancer. Among oncoproteins (E5, E6 and E7) which have a high transformation potential when transduced together. Reactive oxygen species (ROS) constitute a potentially harmful by-product of many cellular reactions, hence an efficient clearance is required. HR-HPVs can adapt to oxidative stress (OS) conditions by upregulating the formation of endogenous antioxidants such as catalase, glutathione (GSH) and peroxiredoxin (PRX). The main aim of this work was to study how these oncoproteins cooperate to boost key cancer cell features such as uncontrolled cell proliferation, invasion potential, and oxidative lines, expressing the following combination of oncogenes: E5-18 E6/E7-18 and E5/E6/E7-18 Oen results showed a statistically significant increment in cell viability, proliferation in the cell line containing the three oncogenes. The highest invasion potential, and expression of two proangiogenic proteins (U-PA,CapG) was observed in HaCaT E5/E6/E7-18 cells. Additionally, we assessed the levels
(u) of three systems involved in ROS detoxification and noticed that cells carrying HPV-18 E5/E6/ET presented a decrease in catalase activity and a significant augmentation of GSH, PRX1 and HO-
$1 / H M O X 1$ levels relative to E5, E6/E7, and HaCaT cells. In conclusion, this study demonstrates that the three HPV-18 E5, E6 and E7 oncoproteins can cooperate to enhance the malignant transformation of HaCaT cells.

\section{MATERIAL AND METHODS}

Transduction of HPV-18 E5,E6 and E7 in spontaneously immortalized human keratinocytes (HaCaT). HaCaT E5/E6/E7 cells were obtained by co-infection with a lentiviral vector carrying the MSCV-N-puro$18 E 5$ plasmid, and with a pLXSN retroviral vector that contained cloned HPV-18 E6/E7 genes. $15 \mathrm{\mu g}$ of each plasmid were used to transfect the packaging ecotropic Bosc23 cells using the FUGENE $\Theta 6$ Transfection
Reagent (Promega, Wisconsin, USA). After $48 \mathrm{~h}$, cell supernatants were used to transduce amphotropic Am-12 cells in order to obtain high-titer retroviral supernatants in the presence of $10 \mathrm{mg} \mathrm{mL}-1$ polybrene. At $48 \mathrm{~h}$ post infection, $\mathrm{HaCaT}$ cells infected with retroviral vector containing E6/E7 HPV-18 were selected with $0.5 \mathrm{mg} \mathrm{mL}-1 \mathrm{G} 418$ (Gibco, Massachusetts, USA), and cells infected with retroviral vector containing E5
HPV-18 were selected with $0.5 \mu \mathrm{mL}-1$ of Puromycin (Santa Cruz Biotechnology, Texas, USA). Viral stocks were titrated by a NIH3T3 cells G418-resistant colony assay. Equal amounts of virus were used to infect
with HaCaT cells (at MOI=10). Total cellular RNA was extracted using TRIzol@ (Life Technologies, California, USA). CDNAs were generated by reverse transcription from $2.000 \mathrm{ng}$ of total RNA in a total volume of 20 $\mu L$ using the High Capacity RNA to CDNA kit (Life Technologies, California, USA). CDNAs (100 ng $\mu$ L-1), CDNA samples of cells transduced with HPV-18E5, HPV-18E6/E7, and HPV-18 E5/E6/E7, were subjected to PCR amplification with specific primers for HPV-18 E5, and E7 amplicons

Viability and proliferation assays. Cell viability was assessed using 3-(4,5-dimethylthiazol-2-yl)-2,5diphenyl tetrazolium bromide) (MTT). In this assay, cell (Sigma-Aldrich, Missouri, USA). After 4 hours, $100 \mu \mathrm{LL}$ of dimethylsulfoxide (DMSO) was added to each well to dissolve the formazan crystals. Finally, the absorbance was measured at $570 \mathrm{~nm}$ in a Varioskan FLASH equipment (Thermo Fischer Scientific, Massachusetts, USA). A cell proliferation curve was performed by counting viable cells in a Neubauer chamber for four days. For this, $5.0 \times 10^{4}$ cells (day 0) of the stable HaCaT lines were plated; HaCaT E5-18; HaCaT E6/E7-18 and HaCaT E5/E6/E7-18 in 6-well plates, eac
plate was numbered corresponding to a different day of the experiment (day 1, day 2, day 3 and day 4). plate was numbered corresponding to a different day of the experiment (day 1, day 2, day 3 and day 4). Invasion assay: The invasiveness potential of HaCaT, HaCaT E5, HaCaT E6/E7, HaCaT E5/E6/E7 was
measured using the $Q C M^{T M}$ Collagen Cell Invasion Assay (Merck Millipore, Darmstadt, Germany) following manufacturer's instructions.

ROS quantification; Intracellular oxidant levels were measured in the four cell lines (HaCaT, HaCaT E5 HaCaT E6/E7, HaCaT E5/E6/E7) using the fluorescent probe 5-(and-6)-chloromethyl-2'-7'dichlorodihydrofluorescein diacetate (CM-H2DCFDA) (Thermo Fischer Scientific, Massachusetts, USA). $C M-H 2 D C F D A$
plate containing $1 \times 10^{4}$ cells per well. Plates were incubated for $30 \mathrm{~min}$ at $37^{\circ} \mathrm{C}$ and cells were analyzed by plate containing $1 \times 10^{4}$ cells per well. Plates were incubated for $30 \mathrm{~min}$ at $37^{\circ} \mathrm{C}$ and cells were analyzed by
flow cytometry, in a BD FACS Calibur cytometer. Catalase activity and GSH quantification: Catalase activity was assayed spectrophotometrically, following
the decomposition of $10 \mathrm{mM} \mathrm{H} H 2 O 2$ by catalase contained in the samples at $240 \mathrm{~nm}(\varepsilon(240 \mathrm{~nm})=39.4 M$ $1 \mathrm{~cm}-1)$. Dilutions of cell lysates were used with concentrations ranging from $0.75 \mu \mathrm{g} \mu \mathrm{L}-1$ to $1.0 \mu \mathrm{g} \mu \mathrm{L}-1$ of total protein in Hank's Balanced Salt Solution (HBSS). Absorbance measurements were conducted at room temperature in a UV-Vis Varian Cary 50 spectrophotometer for 2 minutes or until the absorbance value
decreased by $10 \%$ with respect to the initial rate conditions. Total glutathione in cell lysates was quantified decrease
by HPLC.

PRX1 expression level: HaCaT cell pellets from the four cell lines (HaCaT, HaCaT E5, HaCaT E6/E7, HaCaT E5/E6/E7) were washed with ice cold phosphate buffered saline (PBS), centrifuged, and protein lysates ( (1X) 5\% BSA $0.6 \%$ Tween 20 for ane hoche then incubated with the primary antibody against peroxiredoxin 1 (Anti-PRX1) diluted 1/1000 in TBS (1X) $5 \%$ BSA, $0.1 \%$ Tween 20 (AB Frontier, LF PA0086, Seoul, Korea).

Proteome profiling of keratinocytes transduced with HPV18 oncogenes: Relative intensity of 84 Preferent proteins involved in different signaling mediated pathways related to cancer were evaluated using the Human XL Oncology Array Kit (R\&D Systems, MN, USA, ARYO26)

\section{RESULTS}

\section{ind}

